\title{
Evaluation of Microvascular Permeability with Dynamic Contrast-Enhanced MRI for the Differentiation of Primary CNS Lymphoma and Glioblastoma: Radiologic-Pathologic Correlation
}

P. Kickingereder, F. Sahm, B. Wiestler, M. Roethke, S. Heiland, H.-P. Schlemmer, W. Wick, A. von Deimling, M. Bendszus, and A. Radbruch

\begin{abstract}
BACKGROUND AND PURPOSE: Dynamic contrast-enhanced MR imaging can provide in vivo assessment of the microvasculature in intracranial tumors. The aim of the present study was to evaluate the diagnostic performance of dynamic contrast-enhanced MR imaging derived vascular permeability parameters, including the volume transfer constant, the volume of extravascular extracellular space, and the flux rate constant between the extravascular extracellular space and plasma, for the differentiation of primary CNS lymphoma and glioblastoma.
\end{abstract}

MATERIALS AND METHODS: Sixty glioblastomas and 11 primary central nervous system lymphomas were included. Pretreatment T7weighted dynamic contrast-enhanced MR imaging with a 3D T1-weighted spoiled gradient-echo sequence was performed on a 3T MR imaging scanner. Perfusion parameters (volume transfer constant, the volume of extravascular extracellular space, and the flux rate constant) were measured on the basis of the Tofts-Kernmode model. The Mann-Whitney $U$ test and receiver operating characteristic analysis were used to compare those parameters between primary central nervous system lymphoma and glioblastoma. Histopathologic correlation of dynamic contrast-enhanced MR imaging findings was performed by using reticulin staining and CD31 immunohistochemistry.

RESULTS: Median volume transfer constant and flux rate constant values were significantly higher in primary central nervous system lymphoma $(0.145 \pm 0.057$ and $0.396 \pm 0.088)$ than in glioblastoma $(0.064 \pm 0.021$ and $0.230 \pm 0.058)(P<.001$, respectively). Median volume of extravascular extracellular space values did not differ significantly between primary central nervous system lymphoma (0.434 \pm 0.165$)$ and glioblastoma $(0.319 \pm 0.107)$. On receiver operating characteristic analysis, volume transfer constant had the best discriminative value for differentiating primary central nervous system lymphoma and glioblastoma (threshold, 0.093; sensitivity, 90.9\%; specificity, 95.0\%). Histopathologic evaluation revealed intact vascular integrity in glioblastoma despite endothelial proliferation, whereas primary central nervous system lymphoma demonstrated destroyed vessel architecture, thereby promoting vascular disintegrity.

CONCLUSIONS: Primary central nervous system lymphoma demonstrated significantly higher volume transfer constant and flux rate constant values compared with glioblastoma, implying a higher vascular permeability in primary central nervous system lymphoma. These findings confirm initial observations from perfusion CT and dynamic contrast-enhanced MR imaging studies, correlating with underlying histopathologic features, and may be useful in distinguishing primary central nervous system lymphoma from glioblastoma.

ABBREVIATIONS: DCE-MRI = dynamic contrast-enhanced MRI; GB = glioblastoma; $\mathrm{HE}=$ hematoxylin-eosin; $\mathrm{K}_{\mathrm{ep}}=$ flux rate constant; $K^{\text {trans }}=$ volume transfer constant; $\mathrm{PCNSL}=$ primary central nervous system lymphoma; $\mathrm{V}_{\mathrm{e}}=$ volume of extravascular extracellular space

$\mathbf{P}$ reoperative differentiation of primary central nervous system lymphomas (PCNSLs) and glioblastomas (GBs) is of high clinical relevance because neurosurgical strategies for PCNSL and

Received November 3, 2013; accepted after revision January 9, 2014. From the Departments of Neuroradiology (P.K., S.H., M.B., A.R.), Neuropathology (F.S., A.v.D.), and Neuro-oncology (B.W., W.W.), Neurology Clinic, University of Heidelberg Medical Center, Heidelberg, Germany; and German Cancer Consortium, Clinical Cooperation Unit Neuropathology (F.S., A.v.D.), and Clinical Cooperation Unit Neurooncology (B.W., W.W.), and Department of Radiology (M.R., H.-P.S., A.R.), German Cancer Research Center, Heidelberg, Germany.

Paper previously presented at: European Congress of Radiology, March 6-10, 2014; Vienna, Austria.

Please address correspondence to Philipp Kickingereder, MD, Department of Neuroradiology, University of Heidelberg, Im Neuenheimer Feld 400, 69120 Heidelberg, Germany; e-mail: philipp.kickingereder@med.uni-heidelberg.de

http://dx.doi.org/10.3174/ajnr.A3915
GB favor a biopsy-only versus a maximal safe resection. ${ }^{1,2}$ Dynamic contrast-enhanced (DCE) MR imaging allows characterization of the vascular microenvironment in brain tumors by measurement of a range of parameters that reflect specific physiologic characteristics. ${ }^{3}$ Several recent studies have successfully applied DCE-MR imaging for quantitative estimates of vascular permeability parameters, essentially for glioma grading ${ }^{4-6}$ and response assessment to antiangiogenic therapies. ${ }^{7,8}$ The most widely applied and accepted MR imaging standard to measure microvascular permeability is the DCE steady-state T1weighted method, which is based on the 2-compartment pharmacokinetic model of Tofts and Kermode. ${ }^{9,10}$ Thereby the diffusive transport of low-molecular-weight contrast agent across the capillary endothelium from the blood plasma to the ex- 
travascular extracellular space is represented by the volume transfer constant $\left(K^{\text {trans }}\right.$, minute $\left.{ }^{-1}\right)$. Further parameters considered in this model are the flux rate constant $\left(\mathrm{K}_{\mathrm{ep}}\right.$, minute $\left.^{-1}\right)$ between the extravascular extracellular space and the blood plasma and the volume $\left(\mathrm{V}_{\mathrm{e}}\right)$ of the extravascular extracellular space per volume unit of tissue. ${ }^{3,9}$

Distinct structural and ultrastructural differences in the microenvironment of the tumor vasculature on histologic assessment have been reported for PCNSL and GB, suggesting increased vascular permeability for PCNSL. ${ }^{11}$ However, radiologic comparison of vascular permeability in PCNSL and GB has as yet only been performed with perfusion $\mathrm{CT}^{12,13}$ or with DSC-PWI processed with a mathematic leakage-correction model, ${ }^{14}$ which, in part, confirmed the increased vascular permeability for PCNSL. The aim of our study was to evaluate the diagnostic performance of DCE-MR imaging-derived vascular permeability parameters, including $K^{\text {trans }}, \mathrm{K}_{\mathrm{ep}}$, and $\mathrm{V}_{\mathrm{e}}$ for the preoperative differentiation of PCNSL and GB. Furthermore, histopathologic correlation of DCE-MR imaging findings was performed for all patients by using reticulin and CD31 immunohistochemistry.

\section{MATERIALS AND METHODS \\ Patient Selection}

Our institutional review board approved this retrospective study, and the requirement for patient informed consent was waived. Potentially eligible patients from October 2009 to June 2013 were identified on the basis of the diagnosis of PCNSL or GB. Next, for the selection of appropriate patients with PCNSL, only previously untreated, immunocompetent candidates were included. Thereby, 11 patients with PCNSLs with available DCE-MR imaging data on pretreatment MR imaging were available for inclusion in the present study. For selection of appropriate patients with GB, similar inclusion criteria were used. In detail, only previously untreated patients with the availability of pretreatment DCE-MR imaging data were included. Thereby, a control sample of 60 patients with GB was selected for inclusion in the present study.

\section{MR Imaging}

Images were acquired in the routine clinical work-up by using a 3T MR imaging system (Magnetom Verio/Trio Tim; Siemens, Erlangen, Germany) with a 12-channel head-matrix coil. Axial DCE-MR imaging was performed with a 3D spoiled gradientecho sequence providing coverage of the entire brain by using the following parameters: matrix size, $128 \times 128$; FOV, $172 \times 230$ mm; TR, $5.28 \mathrm{~ms}$; TE, $2.45 \mathrm{~ms}$; flip angle, $10^{\circ}$; section thickness, 5 $\mathrm{mm}$. Thereby, 22 dynamic acquisitions, each including $26 \mathrm{sec}-$ tions, were obtained every 13.34 seconds, resulting in a total measurement time of 4:53 minutes. After the third dynamic acquisition, a standard dose $(0.1 \mathrm{mmol} / \mathrm{kg}$ body weight $)$ of gadoterate meglumine (Dotarem; Guerbet, Aulnay-sous-Bois, France) was injected as a bolus through a pneumatically driven injection pump at an injection rate of $5 \mathrm{~mL} / \mathrm{s}$. After completion of the DCE-MR imaging sequence, standard postcontrast MPRAGE data were acquired with $\mathrm{TI}=1100 \mathrm{~ms}, \mathrm{TE}=4 \mathrm{~ms}$, TR $=1710 \mathrm{~ms}$, and flip angle $=15^{\circ}$.

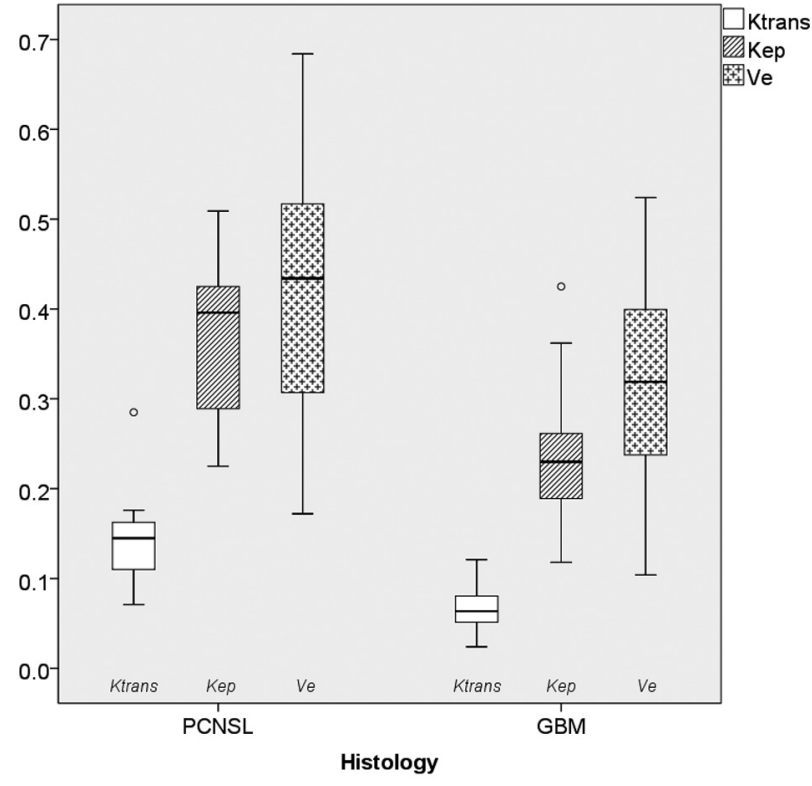

FIG 1. Boxplots of microvascular permeability parameters in primary CNS lymphoma and glioblastoma, including the volume transfer constant (minute ${ }^{-1}$ ), the volume of extravascular extracellular space per volume unit of tissue, and the flux rate constant between extravascular extracellular space and plasma (minute ${ }^{-1}$ ). The solid line inside the box represents the median value, while the edges represent the 25th and 75th percentiles. The straight line (bar) on each box indicates the range of data distribution. Circles represent outliers (values $>1.5$ box length from the 75th and 25th percentile).

\section{Image Postprocessing and Analysis}

Postprocessing of DCE-MR imaging data was performed with dedicated postprocessing software (Tissue 4D; Siemens) based on the Tofts-Kermode model. ${ }^{9}$ First, motion correction was performed on the dynamic images on the basis of an elastic 3D registration technique. Signal intensity was then converted to gadoterate meglumine concentration, and the time-signal intensity curve was calculated. A value for the arterial input function was determined automatically on the basis of a mathematic simulation as described by Orton et al. ${ }^{15}$ After obtaining a kinetic modeling parameter map, a radiologist who was blinded to the patient's histology placed freehand ROIs within the entire contrast-enhancing tumor on each section of the DCE-MR images. Cystic or necrotic regions and normal vessels were avoided during region-of-interest placement. Following delineation of the entire contrast-enhancing tumor, vascular permeability parameters $\left(K^{\text {trans }}, \mathrm{K}_{\mathrm{ep}}\right.$, and $\mathrm{V}_{\mathrm{e}}$ ) were automatically generated on the basis of the pixel information from all placed freehand ROIs.

\section{Histopathologic Correlation}

To assess whether DCE-MR imaging-derived vascular permeability parameters correlate with vascular integrity on histopathology, we performed reticulin (Tibor-Pap) and hematoxylin-eosin (HE) staining of formalin-fixed paraffin-embedded tumor tissue for all patients. Thereby, both HE and Tibor-Pap stains were used to illustrate the architecture of the tumor and vessels, whereas Tibor-Pap highlights the reticulin fibers in the outer border of the vessel wall, which may be destroyed and detached from the inner endothelium membrane in case of decreased vascular integrity. ${ }^{16}$ 

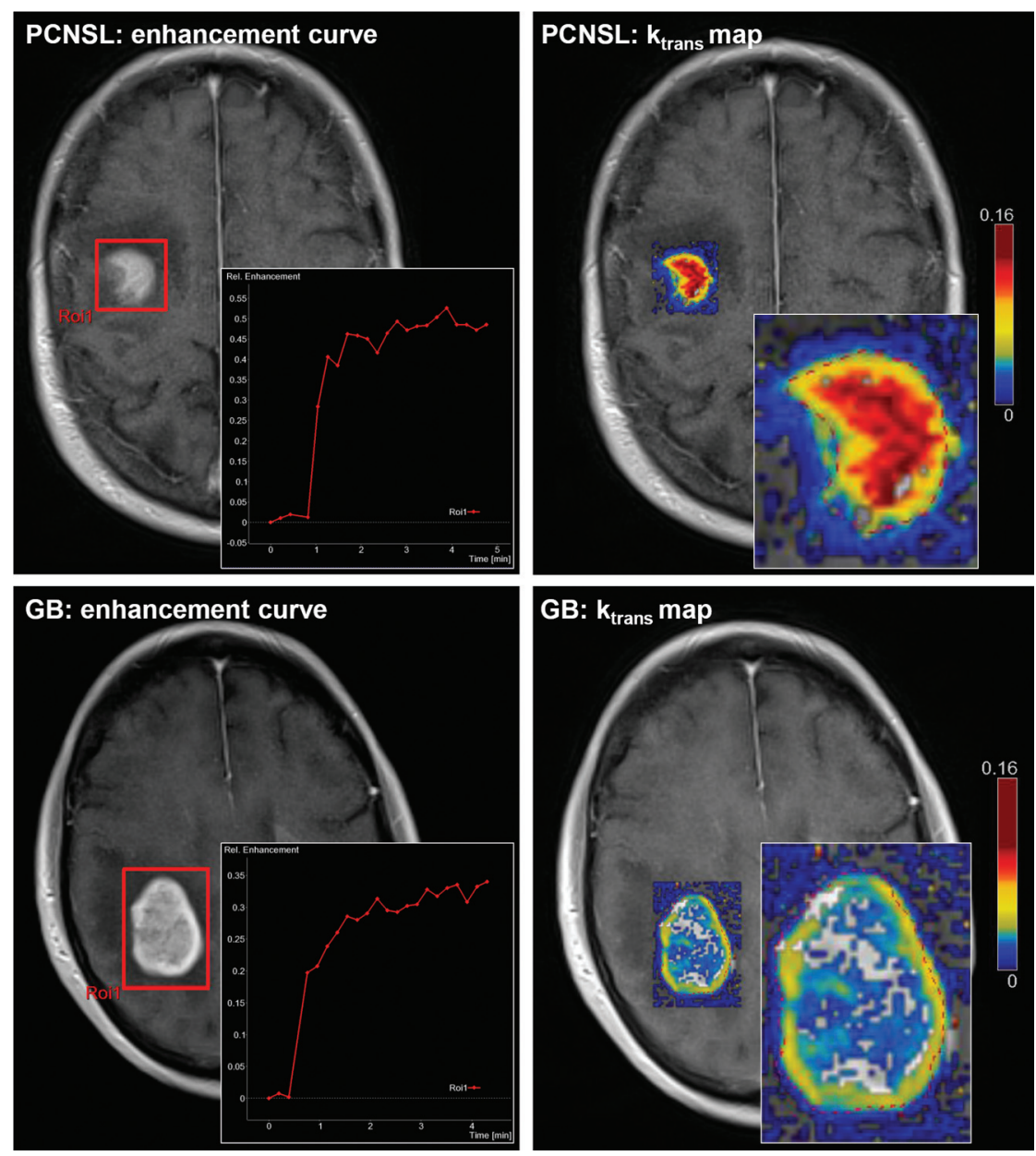

FIG 2. Tissue concentration of contrast agent after bolus injection of $0.1 \mathrm{mmol} / \mathrm{g}$ of gadoterate meglumine (left column) in primary CNS lymphoma (upper row) and glioblastoma (lower row). Note the differences in the relative enhancement curve within the region of interest, encompassing the tumor (red square), which peaks at 0.53 in PCNSL and at 0.34 in GB. Perfusion parameters from kinetic modeling parameter maps (right column) were measured by using freehand region-of-interest placement within the solid part of the entire contrast-enhancing tumor (dashed red line within the zoomed kinetic maps; only 1 section is shown here). In these samples, median $K^{\text {trans }}$ values were $0.147 \pm 0.031$ for PCNSL and $0.045 \pm 0.014$ for GB.

\section{Statistical Analysis}

Statistical analysis was performed by using the Statistical Package for the Social Sciences Statistics, Version 20.0 (IBM, Armonk, New York). A Mann-Whitney $U$ test was used to compare vascular permeability parameters $\left(K^{\text {trans }}, \mathrm{K}_{\mathrm{ep}}\right.$, and $\mathrm{V}_{\mathrm{e}}$ ) between PCNSL and GB. Receiver operating characteristic analysis was performed to determine the optimum thresholds for discrimination of PCNSL and GB. $P$ values $<.05$ were considered significant.

\section{RESULTS}

Figure 1 shows a boxplot of DCE-MR imaging-derived vascular permeability parameters, including $K^{\text {trans }}, \mathrm{K}_{\mathrm{ep}}$, and $\mathrm{V}_{\mathrm{e}}$. Median $K^{\text {trans }}$ values were higher in PCNSL $(0.145 \pm 0.057$; 95\% CI, $0.106-$ 0.183 ; range, $0.071-0.285)$ than in $\mathrm{GB}$ (0.064 $\pm 0.021 ; 95 \%$ CI, 0.060-0.071; range, $0.024-0.121)(P<.001)$. Similarly, median $\mathrm{K}_{\mathrm{ep}}$ values were higher in PCNSL (0.396 土 0.088; 95\% CI, 0.3050.423 ; range, $0.225-0.509)$ than in $\mathrm{GB}$ $(0.230 \pm 0.058 ; 95 \% \mathrm{CI}, 0.215-0.245$; range, $0.118-0.425)(P<.001)$. Median $\mathrm{V}_{\mathrm{e}}$ values did not differ significantly between PCNSL $(0.434 \pm 0.165 ; 95 \% \mathrm{CI}$, $0.311-0.534$; range, $0.172-0.684)$ and GB (0.319 \pm 0.107; 95\% CI, 0.292-0.347; range, $0.104-0.524)$. On receiver operating characteristic analysis, $K^{\text {trans }}$ had the best discriminative value for differentiating PCNSL and GB (area under the curve, 95.6\%; threshold, 0.093; sensitivity, 90.9\%; specificity, 95.0\%) compared with $\mathrm{K}_{\mathrm{ep}}$ (area under the curve, $89.1 \%$; thresh-

Additionally, in cases in which the endothelial architecture was not evident from HE and reticulin staining, CD31 immunohistochemistry, highlighting endothelial cells, was performed. Tissue was provided by the Department of Neuropathology, Institute of Pathology, University of Heidelberg Medical Center, Germany, in accordance with local ethics approval. Sections cut to $3 \mu \mathrm{m}$ were processed by using a BenchMark XT immunostainer (Ventana Medical Systems, Tucson, Arizona). Reticulin and HE staining was performed by applying standard protocols. Pretreatment for antigen retrieval was followed by incubation with antihuman CD31 antibody (Dako, Glostrup, Denmark). Incubation was followed by Ventana standard signal amplification, UltraWash, counter-staining with 1 drop of hematoxylin for 4 minutes and 1 drop of bluing reagent for 4 minutes. For visualization, the UltraView Universal DAB Detection Kit (Ventana Medical Systems) was used. Reticulin, HE, and CD31 stains were analyzed by a neuropathologist by using a Olympus BX-50 microscope (Olympus America, Lake Success, New York), and vascular integrity was rated as intact or decreased. old, 0.272; sensitivity, 90.9\%; specificity; $78.3 \%$ ) or $\mathrm{V}_{\mathrm{e}}$ (area under the curve, $68.6 \%$; threshold, 0.410 ; sensitivity, $63.6 \%$; specificity, $76.7 \%)$. The workflow of postprocessing DCE-MR imaging data of 2 representative patients with PCNSL and GB is shown in Fig 2.

On histopathologic assessment of vascular integrity, tissue samples from PCNSL demonstrated destroyed vessel architecture in each case. Thereby, the outer border of the vessel wall was infiltrated by tumor cells (ie, angiocentric growth pattern), destroyed, and detached from the inner endothelial membrane, thereby contributing to vascular disintegrity (Fig 3, left column). In contrast, vascular integrity was rated as intact for all GBs (Fig 3, right column).

The present study evaluated the diagnostic performance of DCE-MR imaging-derived vascular permeability parameters to facilitate the preoperative differentiation of PCNSL and GB. As a principal finding, our study shows that PCNSL demonstrates significantly higher $K^{\text {trans }}$ and $\mathrm{K}_{\mathrm{ep}}$ values on DCE-MR imaging com-

\section{DISCUSSION}



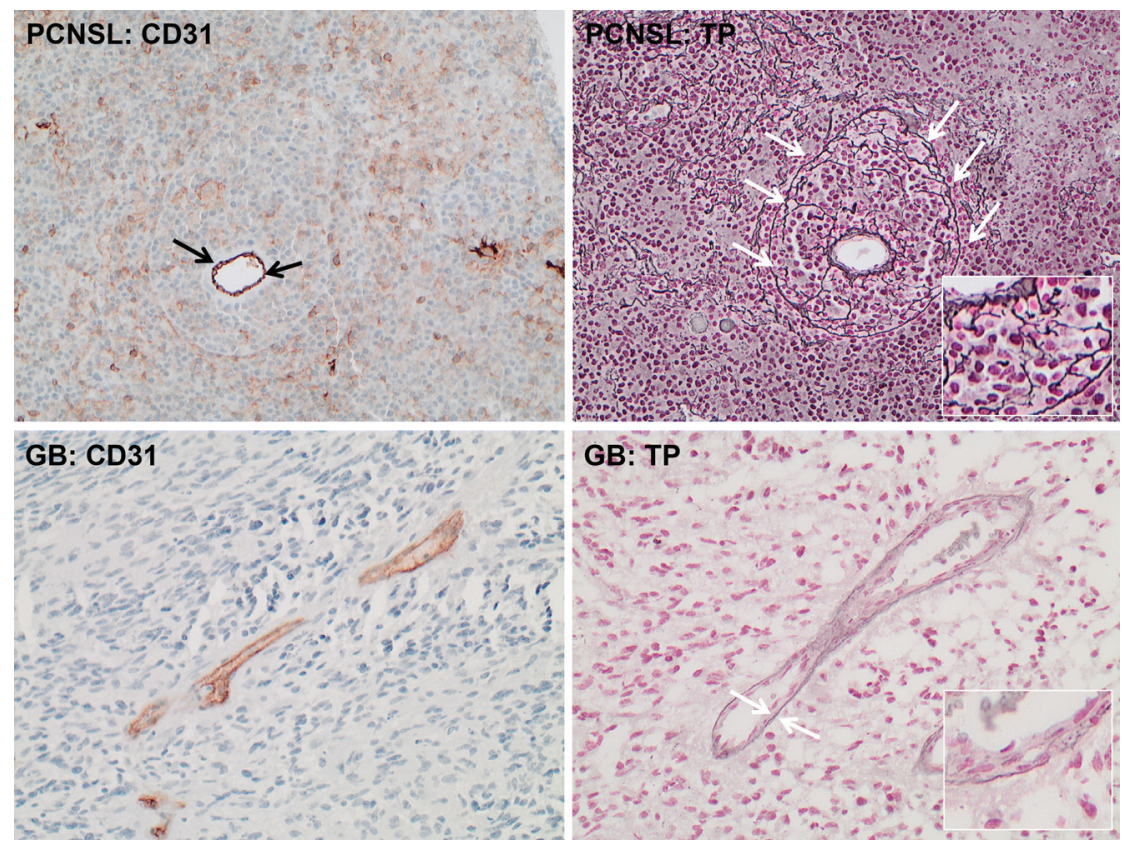

FIG 3. Histopathologic assessment of vascular integrity from those patients shown in Fig 2 (original magnification $\times 200$ for all stains): Immunohistochemistry for CD31 (left column) highlights endothelium in primary CNS lymphoma (PCNSL) (upper row) and glioblastoma (GB) tissue (lower row). Black arrows point to the endothelium and indicate the actual lumen of the vessel in PCNSL tissue. In contrast, white arrows in the reticulin staining of the PCNSL tissue (upper row, right) illustrate the outer border of the vessel wall, which is infiltrated by tumor cells, destroyed, and detached from the inner endothelial membrane and thus contributing to decreased vascular integrity. White arrows in the reticulin staining of the GB tissue (lower row, right) underline the integer basal membrane of vessels despite endothelial proliferation.

pared with GB, thereby implying a greater degree of BBB disruption and thus higher vascular permeability in PCNSL, ultimately confirming initial observations from perfusion CT and DSC-MR imaging studies. ${ }^{13,14}$ Furthermore, we could demonstrate that these findings correlate with underlying histopathologic features, with intact vascular integrity for GB and destroyed vessel architecture in PCNSL, thereby promoting vascular disintegrity in the latter group.

Radiologic comparison of vascular permeability patterns between PCNSL and GB was initially performed by Warnke et al, ${ }^{13}$ who demonstrated significantly higher $K^{\text {trans }}$ values on CT perfusion imaging for PCNSL compared with GB. These results were in contrast to a subsequent study by Schramm et al, ${ }^{12}$ who performed CT perfusion imaging in a series of 31 high-grade gliomas and 8 PCNSLs, reporting no significant difference in $K^{\text {trans }}$ values on Patlak analysis. This pharmacokinetic model assumes that the transfer of the contrast agent across the BBB is unidirectional from the blood plasma to the extravascular extracellular space throughout the entire measurement. ${ }^{17}$ In contrast, the equation of Tofts and Kermode ${ }^{9}$ also considers the backflow of contrast agent from the extravascular extracellular space to the blood plasma. However, neglecting the backflow of the contrast agent in the Patlak model essentially underestimates $K^{\text {trans }}$ in brain tumors ${ }^{18-20}$ and may explain the nonsignificant differences in the study of Schramm et al. ${ }^{12}$

Recently, Toh et al ${ }^{14}$ performed a study on 15 patients with PCNSL and 20 with GB, evaluating the pseudoleakage parameter $\mathrm{K}_{2}$ derived from DSC-PWI with a mathematic leakage-correction model, reporting significant higher $K_{2}$ values in PCNSL compared with GB. This model is based on the assumption that in brain tumors with substantial BBB breakdown, contrast agent extravasates into the extravascular extracellular space, thereby reducing the $\mathrm{T} 2{ }^{\star}$ DSC-PWI signal-intensity loss by signal-intensity increase in regions where $\mathrm{T} 1$ effects are significant. ${ }^{21,22}$ In such instances, CBV will be underestimated. Therefore Boxerman et $\mathrm{al}^{21,22}$ and Weisskoff et $\mathrm{al}^{23}$ proposed a mathematic leakage-correction model in which linear fitting is used to determine the voxelwise deviation from nonleaky reference tissue and, by removing the leakage term, allowing generation of both corrected CBV maps and first-order estimates of vascular permeability. Although direct comparison is limited by differences in study populations and methodology, the diagnostic performance of DCE-derived $K^{\text {trans }}$ in the present study was superior compared with the mathematic leakage-corrected DSC-derived $K_{2}$ in the study of Toh et $\mathrm{al}^{14}$ (area under the curve on receiver operating characteristic analysis, $95.6 \%$ versus $77.1 \%)$. Due to the complex relationship between contrast agent concentration in tissue and the measured change in signal intensity on DSC-PWI, a positive relationship between DSC-PWI and DCE-MR imaging-derived vascular permeability parameters (ie, $K_{2}$ and $\left.K^{\text {trans }}\right)$ may only exist with high flip angles on DSCPWI $\left(90^{\circ}\right) .^{24}$

Furthermore, the DSC-PWI leakage-correction model assumes that the contrast agent arrives at the same time in the tumor and unaffected normal brain tissue and that tumor and nontumor tissue have equal MTT. ${ }^{25,26}$ However, tumor vasculature is extremely variable and heterogeneous, ${ }^{27}$ and elevated tumor MTT may cause overestimation of $\mathrm{CBV}$ and underestimation of $K_{2}{ }^{22,26}$ Therefore, Bjornerud et $\mathrm{al}^{26}$ recently described a novel leakagecorrection algorithm for evaluating microvascular permeability on DSC-PWI that is insensitive to MTT. However, the clinical value of such mathematically leakage-corrected DSC-PWI permeability parameters has to be determined in future studies and correlated with those from DCE-MR imaging.

Previous studies have demonstrated that CBV values significantly differ between PCNSL and GB, with only modest increased values in the former and markedly increased values in the latter group. ${ }^{14,28-30}$ These results may be explained by the differences in neovascularization, which is a hallmark in $\mathrm{GB},{ }^{31}$ however, an unusual feature in (non-AIDS-related) PCNSL. ${ }^{32}$ Thus, the microvascular blood flow to the tumor tissue in GB is often hampered by an abnormal tumor vasculature due to neovascularization comprising immature or defective endothelium, tortuosity, and thrombosis. Hence, the uptake of contrast agent by the GB tissue is likely limited by blood flow. ${ }^{10}$ In contrast, the absence of neovascularization and the substantial disruption of the BBB in 
PCNSL is related to the clustering of tumor cells within and around pre-existing brain vessels (ie, angiocentric growth pattern). ${ }^{33}$ These distinct morphologic differences may make the uptake of contrast agent in PCNSL less flow-limited compared with $\mathrm{GB}$, which may serve as an explanation for the significantly higher $K^{\text {trans }}$ and $K_{\text {ep }}$ values in PCNSL in the present study.

A potential limitation of the present study is that due to the given section thickness of the DCE-MR imaging sequence, volume-averaging effects may have limited exact region-of-interest placement for precise delineation of the contrast-enhancing tumor. Another limitation is that DCE-MR imaging-derived vascular permeability parameters may be affected by several factors, including blood flow; blood volume; endothelial permeability; endothelial permeability surface area; and hydrostatic, interstitial, and osmotic gradients across the endothelium. ${ }^{10}$ Thus, we could not definitely state whether changes in $K^{\text {trans }}$ and $\mathrm{K}_{\mathrm{ep}}$ were solely due to changes in vascular permeability or to what extent other factors may have contributed.

\section{CONCLUSIONS}

PCNSL demonstrated significantly higher $K^{\text {trans }}$ and $K_{\text {ep }}$ values compared with GB, thereby implying a higher vascular permeability in PCNSL. These findings correlate with underlying histopathologic features, with intact vascular integrity for GB and destroyed vessel architecture in PCNSL, thereby promoting vascular disintegrity in the latter group, and they may improve the preoperative differentiation of PCNSL and GB.

Disclosures: Heinz-Peter Schlemmer-UNRELATED: Consultancy: Siemens, Grants/ Grants Pending: Siemens, ${ }^{*}$ Payment for Lectures (including service on Speakers Bureaus): Siemens, Curagita, Covidien; Travel/Accommodations/Meeting Expenses Unrelated to Activities Listed: Siemens. Wolfgang Wick-UNRELATED: Consultancy: Roche, Merck Sharp \& Dohme, Payment for Lectures (including service on Speakers Bureaus): Roche, Prime Oncology, Patents (planned, pending or issued): IDH diagnostic antibody. Martin Bendszus - UNRELATED: Board Membership: Vascular Dynamics, Payment for Lectures (including service on Speakers Bureaus): Novartis, Roche, Codman, Guerbet. *Money paid to the institution.

\section{REFERENCES}

1. Stupp R, Mason WP, van den Bent MJ, et al. Radiotherapy plus concomitant and adjuvant temozolomide for glioblastoma. $N$ Engl J Med 2005;352:987-96

2. Schlegel U. Primary CNS lymphoma. Ther Adv Neurol Disord 2009;2:93-104

3. Tofts PS. T1-weighted DCE imaging concepts: modelling, acquisition and analysis. MAGNETOM Flash 2010;30-39

4. Nguyen TB, Cron GO, Mercier JF, et al. Diagnostic accuracy of dynamic contrast-enhanced MR imaging using a phase-derived vascular input function in the preoperative grading of gliomas. AJNR Am J Neuroradiol 2012;33:1539-45

5. Cha S, Yang L, Johnson G, et al. Comparison of microvascular permeability measurements, $\mathrm{K}^{\text {trans }}$, determined with conventional steady-state T1-weighted and first-pass T2*-weighted MR imaging methods in gliomas and meningiomas. AJNR Am J Neuroradiol 2006;27:409-17

6. Mills SJ, Patankar TA, Haroon HA, et al. Do cerebral blood volume and contrast transfer coefficient predict prognosis in human glioma? AJNR Am J Neuroradiol 2006;27:853-58

7. Sorensen AG, Batchelor TT, Zhang WT, et al. A “vascular normalization index" as potential mechanistic biomarker to predict survival after a single dose of cediranib in recurrent glioblastoma patients. Cancer Res 2009;69:5296-300

8. Zhang W, Kreisl T, Solomon J, et al. Acute effects of bevacizumab on glioblastoma vascularity assessed with DCE-MRI and relation to patient survival. In: Proceedings of the 17th Scientific Meeting and Exhibition of the International Society for Magnetic Resonance in Medicine, Honolulu, Hawaii; April 18-24, 2009:282

9. Tofts PS, Kermode AG. Measurement of the blood-brain barrier permeability and leakage space using dynamic MR imaging. 1. Fundamental concepts. Magn Reson Med 1991;17:357-67

10. Cha $S$. Update on brain tumor imaging: from anatomy to physiology. AJNR Am J Neuroradiol 2006;27:475-87

11. Molnár PP, O’Neill BP, Scheithauer BW, et al. The blood-brain barrier in primary CNS lymphomas: ultrastructural evidence of endothelial cell death. Neuro Oncol 1999;1:89-100

12. Schramm P, Xyda A, Klotz E, et al. Dynamic CT perfusion imaging of intra-axial brain tumours: differentiation of high-grade gliomas from primary CNS lymphomas. Eur Radiol 2010;20:2482-90

13. Warnke PC, Timmer J, Ostertag CB, et al. Capillary physiology and drug delivery in central nervous system lymphomas. Ann Neurol 2005;57:136-39

14. Toh CH, Wei KC, Chang CN, et al. Differentiation of primary central nervous system lymphomas and glioblastomas: comparisons of diagnostic performance of dynamic susceptibility contrast-enhanced perfusion MR imaging without and with contrast-leakage correction. AJNR Am J Neuroradiol 2013;34:1145-49

15. Orton MR, d'Arcy JA, Walker-Samuel S, et al. Computationally efficient vascular input function models for quantitative kinetic modelling using DCE-MRI. Phys Med Biol 2008;53:1225-39

16. Louis DN, Ohgaki H, Wiestler OD, et al. The 2007 WHO classification of tumours of the central nervous system. Acta Neuropathol 2007;114:97-109

17. Patlak CS, Blasberg RG, Fenstermacher JD. Graphical evaluation of blood-to-brain transfer constants from multiple-time uptake data. J Cereb Blood Flow Metab 1983;3:1-7

18. Bagher-Ebadian H, Jain R, Nejad-Davarani SP, et al. Model selection for DCE-T1 studies in glioblastoma. Magn Reson Med 2012;68:241-51

19. Bergamino M, Saitta L, Barletta L, et al. Measurement of blood-brain barrier permeability with T1-weighted dynamic contrast-enhanced MRI in brain tumors: a comparative study with two different algorithms. ISRN Neurosci 2013;2013:6

20. Jain R, Bagher-Ebadian H, Narang J, et al. Optimal period of linearity using Patlak analysis in brain tumors. In: Proceedings of the 18th Scientific Meeting and Exhibition of the International Society for Magnetic Resonance in Medicine, Stockholm, Sweden; May 1-7, 2010:4090

21. Boxerman J, Schmainda K, Weisskoff R. Relative cerebral blood volume maps corrected for contrast agent extravasation significantly correlate with glioma tumor grade, whereas uncorrected maps do not. AJNR Am J Neuroradiol 2006;27:859-67

22. Boxerman JL, Prah DE, Paulson ES, et al. The role of preload and leakage correction in gadolinium-based cerebral blood volume estimation determined by comparison with MION as a criterion standard. AJNR Am J Neuroradiol 2012;33:1081-87

23. Weisskoff RM, Boxerman JL, Sorensen AG, et al. Simultaneous blood volume and permeability mapping using a single Gd-based contrast injection. In: Proceedings of the 2nd Scientific Meeting and Exhibition of the International Society for Magnetic Resonance in Medicine, San Francisco, California; August 6-12, 1994

24. Emblem K, Mouridsen K, Borra R, et al. Does DSC-derived CA extravasation correlate with DCE Ktrans? In: Proceedings of the 19th International Society of Magnetic Resonance in Medicine, Montreal, Quebec, Canada; May 6-13, 2011

25. Emblem KE, Bjornerud A, Mouridsen K, et al. T(1)- and T(2)(*)dominant extravasation correction in DSC-MRI. Part II. Predicting patient outcome after a single dose of cediranib in recurrent glioblastoma patients. J Cereb Blood Flow Metab 2011;31:2054-64

26. Bjornerud A, Sorensen AG, Mouridsen K, et al. T1- and T2*-dominant extravasation correction in DSC-MRI: part I-theoretical considerations and implications for assessment of tumor hemodynamic properties. J Cereb Blood Flow Metab 2011;31:2041-53 
27. McDonald DM, Choyke PL. Imaging of angiogenesis: from microscope to clinic. Nat Med 2003;9:713-25

28. Hartmann M, Heiland S, Harting I, et al. Distinguishing of primary cerebral lymphoma from high-grade glioma with perfusionweighted magnetic resonance imaging. Neurosci Lett 2003;338: $119-22$

29. Wang S, Kim S, Chawla S, et al. Differentiation between glioblastomas, solitary brain metastases, and primary cerebral lymphomas using diffusion tensor and dynamic susceptibility contrast-enhanced MR imaging. AJNR Am J Neuroradiol 2011;32:507-14

30. Liao W, Liu Y, Wang X, et al. Differentiation of primary central nervous system lymphoma and high-grade glioma with dynamic susceptibility contrast-enhanced perfusion magnetic resonance imaging. Acta Radiol 2009;50:217-25

31. Wong ET, Brem S. Antiangiogenesis treatment for glioblastoma multiforme: challenges and opportunities. J Natl Compr Canc Netw 2008;6:515-22

32. Lister A, Abrey LE, Sandlund JT. Central nervous system lymphoma. Hematology Am Soc Hematol Educ Program 2002:283-96

33. Schlegel U, Hochberg F. Primary CNS lymphoma. In: Tonn JC, Westphal M, Rutka JT, et al, eds. Neuro-Oncology of CNS Tumors. Heidelberg, Germany: Springer-Verlag; 2006:292-302 